\title{
Spontaneous neuronal unit activity in the primate basal ganglia and the effects of precentral cerebral cortical ablations
}

\author{
J. Wayne Aldridge, Sid Gilman and George Dauth \\ Department of Neurology, University of Michigan, Ann Arbor, MI 48104 (U.S.A.)
}

(Accepted 10 October 1989)

Key words: Putamen; Caudate nucleus; Neostriatum; Single unit activity; Primate; Motor cortex

\begin{abstract}
The discharge properties of single neuronal units in the putamen, caudate nucleus, and globus pallidus were studied in awake primates. The effects of restricted deafferentation of the striatum were determined by recording single unit activity in animals with unilateral ablation of areas 4 and 6 of Brodmann. The most striking change was on the regularity of unit firing in the putamen. Units in the normal putamen exhibited a wide range of firing rates and variability. In many units discharge rate was very slow. After the lesion, putaminal units discharged in steady spike trains with highly regular patterns of interspike intervals having on average a $63 \%$ reduction in the coefficient of variation. Contrary to expectations, average firing rates actually increased slightly (22\%) from a median value of $4.88 \mathrm{~Hz}$ in controls to $5.95 \mathrm{~Hz}$ in lesioned animals. Although the rates and variability observed in lesioned animals completely overlapped the range of the sample observed in controls, the distributions were shifted such that there were more units with regular discharge patterns and slightly faster firing rates. The caudate nucleus showed no significant change in firing rate or variability. In the globus pallidus, firing rate decreased significantly in the internal segment, and both segments showed an increase in discharge variability. The findings demonstrate that the cerebral cortex strongly influences the spontaneous discharge properties in the basal ganglia. The effects on the variability of spontaneous activity are greater than on the maintenance of tonic firing.
\end{abstract}

\section{INTRODUCTION}

Neurons in the putamen and caudate nucleus receive a large proportion of their afferents from the cerebral cortex. These projections are glutamatergic ${ }^{15,45,71}$ and excitatory $^{28}$, and they are topographically organized. The putamen receives a major proportion of its cortical afferents from the precentral region of the cerebral cortex (areas 4 and 6 of Brodmann) ${ }^{36,61}$. The caudate nucleus, on the other hand, receives a projection from prefrontal association areas ${ }^{27}$ and a smaller quantity of afferents from precentral motor corte ${ }^{36}$. There is a great deal of convergence among cortical contacts in the striatum. Cerebral cortical areas connected with each other also have common termination zones sites in the striatum $^{70}$. Convergence of cortical and subcortical regions can be detected at the level of individual cells in the striatum $^{17,19,32}$. The physiological role of this converging excitatory input is not fully understood. A quantitative analysis and comparison of spontaneous discharge properties in the separate components of the neostriatum and pallidum in unanesthetized primates has not been performed and the contribution of the cortical inputs to regulating cellular activity in these sites is unknown.

The major projection of the neostriatum is to the globus pallidus ${ }^{8,40,46,51,62,69}$ and the principal neurotransmitter of this pathway is $\gamma$-aminobutyric acid (GABA) ${ }^{18}$, $28,44,47,63$. Colocalized peptide transmitters substance $P$ and enkephalins ${ }^{29,35,50}$ also participate in the striatopallidal projection. Electrical stimulation of the neostriatum evokes inhibitory postsynaptic potentials (IPSP's) in the pallidum ${ }^{37,41,49}$ presumably by releasing the inhibitory transmitter GABA. In some studies the IPSP's were found to be preceded by EPSP's ${ }^{37,41}$. The physiological role of the peptides in the striatopallidal pathway remains undetermined. Like the neostriatum, the pallidum has been shown to be modulated in conjunction with movement ${ }^{1,5,7,13,22,59}$.

Ablation of the precentral areas of the cerebral cortex in primates results in a contralateral hemiparesis due to a loss of projections through both corticospinal and cortico-subcortical circuits, including the basal ganglia ${ }^{25}$, 26. Mapping with labelled deoxyglucose reveals a marked decrease of metabolic activity in the neostriatum and pallidum as well as other parts of the nervous system in the first week after the ablation ${ }^{12}$. With time, metabolic activity partially recovers in the caudate nucleus but less well in the putamen ${ }^{24}$. It is presumed that the metabolic changes and subsequent recovery of neurologic function are correlated to the activity of nerve terminals. Thus, a

Correspondence: J. Wayne Aldridge, University of Michigan, 1103 East Huron, Ann Arbor, MI 48104, U.S.A. 
precentral cortical ablation would decrease terminal activity in the putamen and to a lesser degree in the caudate nucleus; however, the level of neuronal activity in the affected terminal fields has not been examined. It is known from other studies that deafferentation of basal ganglia structures alters the rate and pattern of neuronal discharge $^{48,57}$.

This study was initiated to examine systematically the spontaneous discharge properties of single units in the basal ganglia of awake primates. The effects of a precentral cortical ablation on the spontaneous discharge of neostriatal and pallidal neurons was determined to evaluate the influence of the cortical afferents on this circuitry. Our initial hypothesis was that the loss of excitatory terminals following cerebral cortical ablation would result in a decrease of spontaneous neostriatal neuronal activity. It was anticipated that the changes in spontaneous activity would reflect the level of innervation and convergence in the different parts of the striatum and pallidum. We also anticipated finding a concomitant increase in the activity of the globus pallidus due to a disinhibitory release from an anticipated decrease in neostriatal activity.

\section{MATERIALS AND METHODS}

\section{Animals}

Four $M$. fascicularis monkeys, two lesioned animals and two controls, were used. The animals were prepared for chronic recording of single unit activity from the putamen, caudate nucleus, and globus pallidus of the left cerebral hemisphere. Under general anesthesia with i.v. pentobarbital, the animals were placed in a stereotaxic frame and a stainless-steel recording chamber was fixed to the skull. In the lesioned animals, chamber implantation was preceded by the ablation procedure. The procedure began with removal of a bone flap over the cerebral cortex. The dura was reflected and areas 4 and 6 of Brodmann of the left cerebral hemisphere were ablated by subpial resection ${ }^{14,25}$. This ablation technique preserves the underlying white matter, as verified in this laboratory with histological techniques ${ }^{12}$. After the ablation was completed, the dura was sutured in place and the bone flap was fastened to the skull with stainless steel plates and bone screws. The chamber was fixed to the skull by embedding it in dental acrylic in both control and lesioned preparations.

\section{Recording}

To familiarize the animals with the recording procedures, they were brought to the laboratory every day for one month prior to surgery. Spontaneous neuronal activity was recorded while the monkeys were awake and sitting quietly in a primate chair placed inside a sound-restricted. recording booth. A hydraulic microdrive was placed on the recording chamber and used to manipulate tungsten electrodes to the desired recording sites in the basal ganglia. Preamplified neuronal signals were led to an oscilloscope, audio monitor, and tape recorder. Single units were isolated and the neuronal activity was recorded on tape and digitized off-line. In one control monkey, spikes were digitized on-line, making off-line digitization of tape records unnecessary ${ }^{2}$.

Data collection was initiated when visual observations indicated that the animals were not moving. The animals made periodic postural adjustments during the recording sessions, which lasted for several hours. Because unilateral precentral ablation renders the contralateral limbs paretic for long periods of time, the animals were not trained to perform a motor task. Moreover, this study was undertaken to compare neuronal unit activity with metabolic rates $^{12.24}$ under similar conditions, i.e., with the animals sitting quietly in a chair. In some instances, unit activity in controls was modulated by movements of the limbs, trunk, or head, but no attempt was made to analyze these discharges. To further ensure that comparisons of spontaneous unit activity were made only during quiet sitting, recordings were edited off-line to select periods without movement. Recordings of movement-induced noise in a high impedance cable or signals from an accelerometer were recorded to assist off-line editing. The detectors sensed visible movements in the limbs and postural changes in the trunk or head. Spike trains were assessed during periods of quiet sitting, typically 1-3 minutes in duration. The animals did not make continual small movements, e.g. finger tapping, that might elude detection on our movement sensors.

To ensure that recordings were taken when the animals were awake, the animals were observed directly. In addition, EEG activity was recorded in two animals, one control and one lesioned. Wires were connected to the stainless-steel bone screws implanted in the skull. The EEG was visually monitored on an oscilloscope for the presence of large amplitude slow wave signals indicating drowsiness, however, direct visual observations of the animals proved as reliable in detecting drowsiness as monitoring EEG activity.

During recording sessions, control and lesioned animals behaved similarly except for the paresis of the affected limbs. In the two animals in which EEG was monitored, no obvious difference between the lesioned and the control animals was detected in the state of alertness as determined by EEG waveforms. Occasionally, the animals would become drowsy during recording sessions, but there was no systematic difference in this behavior between lesioned animals and controls. All animals made postural adjustments periodically during the recording sessions and no difference was observed in the number of adjustments between the two groups.

To facilitate comparisons with previous studies of brain metabolism after precentral cortical ablation, all recordings were made in the period from 8 to 60 days after the lesion. In control animals recordings were spaced over several months. On the day of sacrifice 6 recording tracks were marked by passing a direct current $(100 \mu \mathrm{A}$ for $30 \mathrm{~s}$ ) through a bipolar steel electrode. The small iron deposits were stained blue by adding $1.5 \%$ potassium ferrocyanide to the $10 \%$ formalin perfusing solution. The brains were frozen, sectioned, and stained with Cresyl violet and Weil stains. Recording tracks were reconstructed and drawn on maps traced from stained sections.

\footnotetext{
Analysis

Digitized spike trains collected during periods of quiet sitting were analyzed in detail off-line. One unit and occasionally two or three units could be detected in each recording. Units were discriminated from each other and from background noise by a stringent procedure comparing the negative peak, positive peak and peak to peak time of each recorded waveform ${ }^{65}$. When two units could be discriminated adequately, both were studied; rarely, three units were discriminable. At some recording sites more than one unit was present but the waveforms were not reliably separated. In these cases the data were not used.

The intervals between spikes were computed and the mean, median and standard deviation were determined for each unit. Interval variability was assessed with the coefficient of variation (mean/S.D.), an index permitting comparisons of units that might have very different mean rates of activity. Interspike interval histograms were computed and plotted for each unit with both $1 \mathrm{~ms}$ and $10 \mathrm{~ms}$ bins. The population statistics were computed and comparisons between control and lesioned animals were made for each structure. The significance of the comparisons was assessed with the Mann-Whitney $U$-test.
} 


\section{RESULTS}

\section{Histological examination}

The extent of the cortical ablation in a lesioned animal is illustrated schematically in Fig. 1. In both animals the underlying white matter remained intact and the most medial portions of the cortex and some areas deep within the sulci were spared by the procedure. The lesion encroached on area 8 and a small part of area 4 was spared.

\section{Spontaneous unit activity}

Recording began 8 days after surgery. Active neostriatal and pallidal units were encountered in the earliest recording sessions in the lesioned animals and units remained active throughout the recording period. Data from totals of 872 units in control animals and 1018 units in lesioned animals were analyzed (Table I). The locations of the recording sites from all 4 animals are shown in Fig. 2 (neostriatum) and Fig. 3 (pallidum).

Putamen. The most conspicuous difference in neuronal discharge between control and lesioned animals was observed in the putamen. Putaminal units in the lesioned animals exhibited a more regular pattern of discharge. Rate plots (Fig. 4) illustrate this increased regularity of

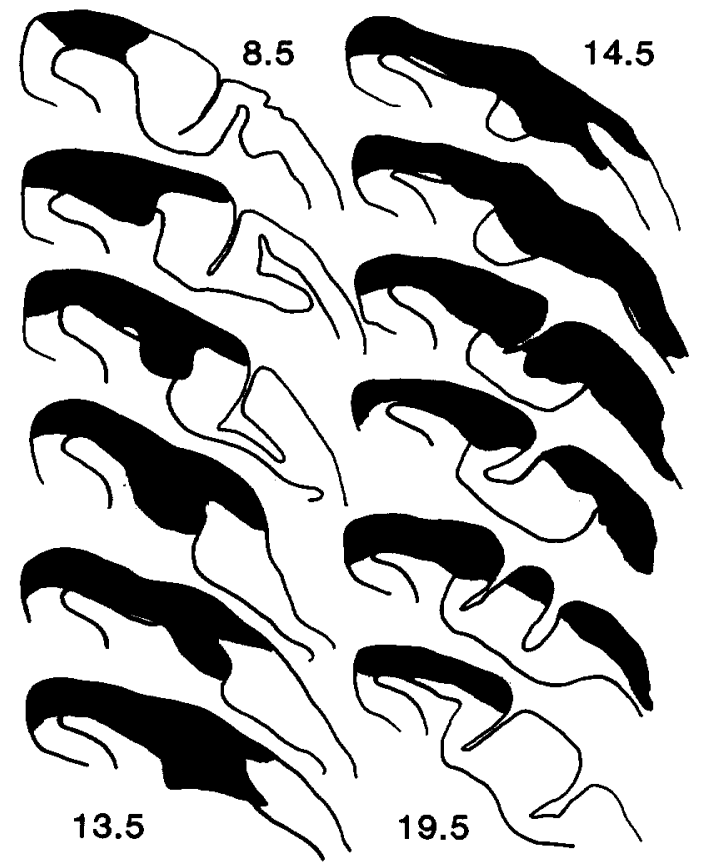

Fig. 1. Schematic illustration of the cerebral cortical ablation. Sections of cerebral cortex, both lesioned and intact sides, were traced from stained sections. The lesioned areas (blackened portions) were marked by inverting and superimposing the tracing of lesioned cortex on the tracing of intact cortex. Sections are separated by $1 \mathrm{~mm}$. The numbers refer to the stereotaxic anteriorposterior coordinates, proceeding in a posterior to anterior direction from the top to the bottom of the figure starting with the left column. The lesion was on the animal's left side.
TABLE I

Interspike intervals and variability

Values are medians (S.E. of median).

\begin{tabular}{lcc}
\hline & Control & Lesion \\
\hline Putamen & & \\
$\quad$ Median (ms) & $205(28)$ & $168(14)^{*}$ \\
Coefficient of variation & $1.53(0.05)$ & $0.96(0.04)^{*}$ \\
Mean rate (Hz) & $1.75(0.19)$ & $3.89(0.18)^{*}$ \\
$n$ & 390 & 519 \\
Caudate nucleus & & \\
$\quad$ Median (ms) & $199(45)$ & $204(36)$ \\
Coefficient of variation & $1.42(0.08)$ & $1.41(0.12)$ \\
Mean rate (Hz) & $1.67(0.32)$ & $2.51(0.40)$ \\
$n$ & 112 & 70 \\
Globus pallidus (lateral) & & \\
$\quad$ Median (ms) & $19(16)$ & $21(19)$ \\
Coefficient of variation & $1.17(0.06)$ & $1.38(0.07)^{*}$ \\
Mean rate (Hz) & $31.3(2.6)$ & $27.8(2.2)$ \\
$n$ & 234 & 293 \\
Globus pallidus (medial) & & \\
$\quad$ Median (ms) & $17(18)$ & $21(5)^{*}$ \\
Coefficient of variation & $1.00(0.14)$ & $1.16(0.05)^{*}$ \\
Mean rate (Hz) & $45.5(4.9)$ & $29.0(2.6)^{*}$ \\
$n$ & 136 & 136 \\
\hline
\end{tabular}

${ }^{*} P<0.001$.
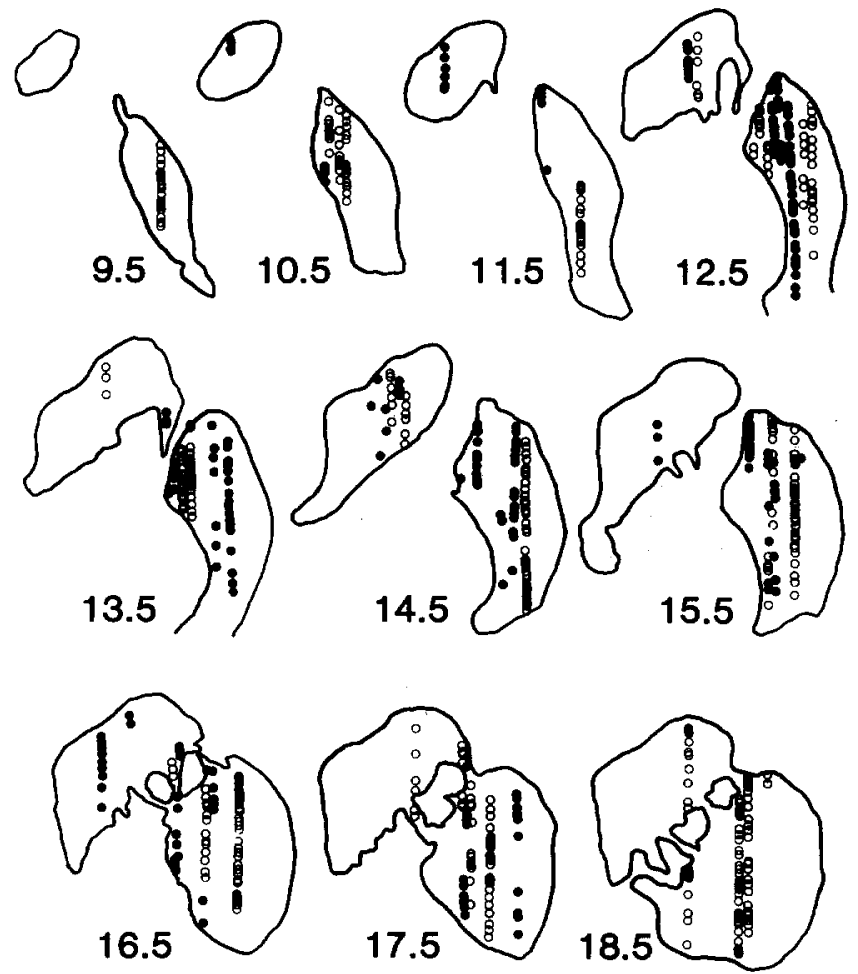

Fig. 2. Neostriatal recording sites. All units are superimposed on sections traced from one animal. Data from controls are plotted as filled circles and from lesioned animals as open circles. The numbers by each section refer to stereotaxic anterior-posterior coordinates. The lesion was on the animal's left side. 
firing. In spike trains from controls, groups of rapidly occurring spikes were separated by periods of silence (Fig. 4A). The silent intervals were often long, producing a characteristic slow discharge pattern in many units. The duration of the silent intervals and the composition of the discharge groups were variable. Long intervals and variable groupings of spikes were not as commonly observed after the lesion (Fig. 4B), instead, the discharge was regular with little fluctuation in rate or interval pattern. Interspike interval histograms (Fig. 5) identified the main features in the interval distribution of spike trains. Interval histograms of control units were highly skewed (Fig. 5A,B), with a small but consistently observed contribution by long interspike intervals. In lesioned animals, these relatively fewer longer interspike intervals were absent; consequently, the interval histograms were less skewed (Fig. 5C). These differences in spike train interval contributions were also evident in the descriptive statistics. The median interspike interval for each distribution, the preferred statistic when data are skewed $^{16}$, was calculated for each spike train. The distributions of median intervals for the entire sample of units are shown in Fig. 6A (control) and Fig. 6C (lesion). In control animals, median intervals ranged from $10 \mathrm{~ms}$ to $3115 \mathrm{~ms}$, or a median discharge rate of $100 \mathrm{~Hz}$ to 0.32
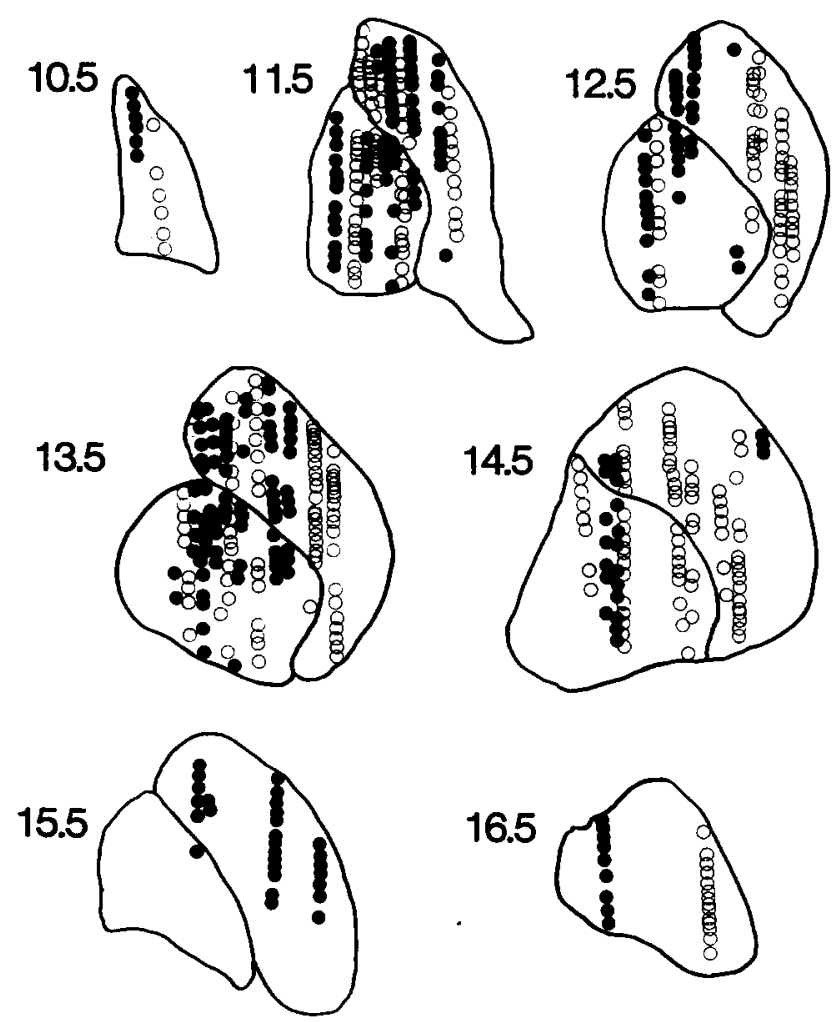

Fig. 3. Pallidal recording sites. All units are superimposed on sections traced from one animal. Data from controls are plotted as filled circles and lesioned animals as open circles. The numbers by each section refer to stereotaxic anterior-posterior coordinates.
$\mathrm{Hz}$. A similar range of values, $10 \mathrm{~ms}$ to $2845 \mathrm{~ms}(100 \mathrm{~Hz}$ to $0.35 \mathrm{~Hz}$ ), was found in lesioned animals, but overall the distribution of median intervals was shifted toward shorter values (Fig. 6A,C). The median intervals in controls and lesioned animals were significantly different $(P<0.001)$. The average (median) for all control units was $205 \mathrm{~ms}$ or a median rate of $4.88 \mathrm{~Hz}$. In lesioned animals, the median was $168 \mathrm{~ms}$ or $5.95 \mathrm{~Hz}$ (Table I). This represents a rate increase of $22 \%$ after the lesion (Fig. 7).

The mean firing rate for each unit was also calculated. Mean putaminal spontaneous discharge rates, as observed in our control sample (Fig. 8), are typically low. It should be noted, however, that the values were distributed in a continuum. There was no obvious division between slow and non-slow units, although this distribution is skewed. For comparative purposes, units with a mean rate less than $3 \mathrm{~Hz}$, a value similar to that used by others ${ }^{4,31,33}$ were arbitrarily categorized as slowly firing. Slow units make up the majority $(69 \%)$ of the
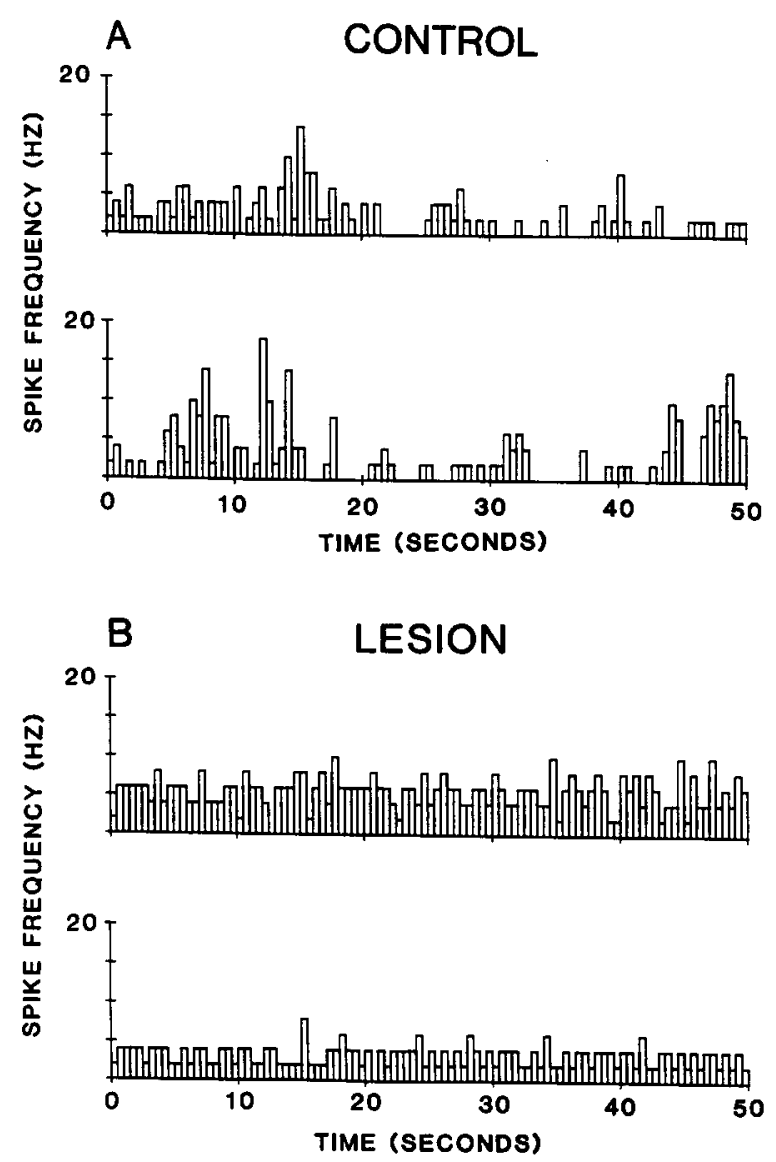

Fig. 4. Rate plots from the putamen. Rate meter plots produced by a computer from the discriminated putaminal spike trains are used to illustrate $50 \mathrm{~s}$ portions of spike trains. The $x$-axis gives the time in seconds and $y$-axis gives the firing rate in spikes per second. Each bin is $500 \mathrm{~ms}$ wide. In A one sample from each control animal is plotted. In B one sample from each lesioned animal is shown. The regularity of firing increased after the lesion. 
A CONTROL

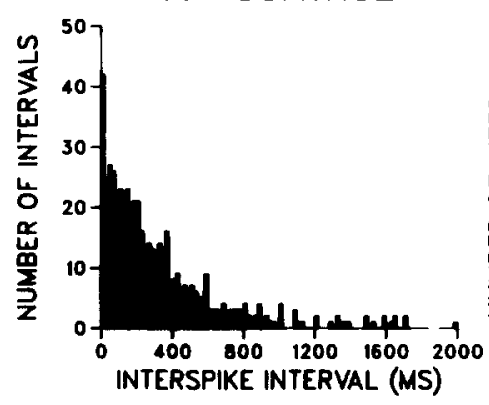

B CONTROL

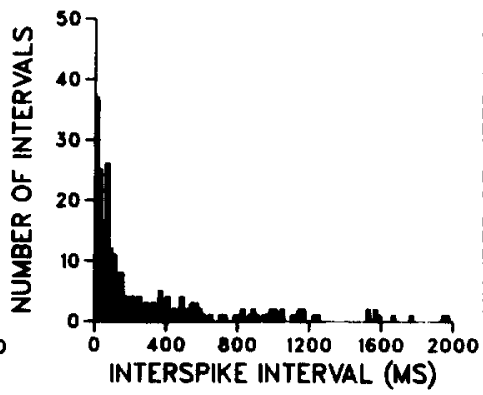

C LESION

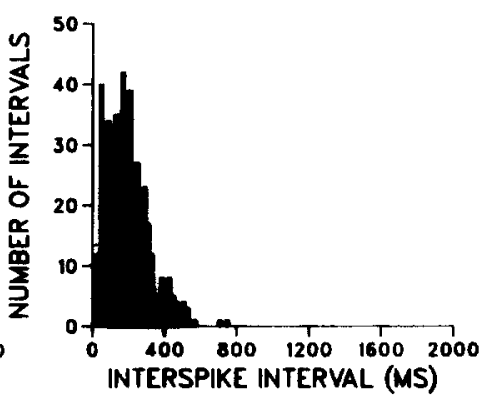

Fig. 5. Interspike interval histograms of putamen units in a control (A and B) and a lesioned animal (C). Each bin has a duration of $20 \mathrm{~ms}$. A: control: median $=217 \mathrm{~ms}(4.6 \mathrm{~Hz})$, coefficient of variation $(\mathrm{CV})=1.28, n=485$ intervals, 5 intervals were greater than $2000 \mathrm{~ms}$ and not plotted. B: control: median $=125 \mathrm{~ms}(8.0 \mathrm{~Hz}), \mathrm{CV}=2.13, n=262$ intervals, 26 intervals were greater than $2000 \mathrm{~ms}$. C: lesioned animal: median $=179 \mathrm{~ms}(5.5 \mathrm{~Hz}), \mathrm{CV}=0.59, n=518$ intervals, all less than $2000 \mathrm{~ms}$.

sample in controls. In lesioned animals, this category made up $40 \%$ of the sample. The average firing rate in controls was $1.75 \mathrm{~Hz}$ in controls and $3.89 \mathrm{~Hz}$ in lesioned animals. Although the latter is a comparatively low firing rate, it represents an increase of $112 \%$ after the ablation.

Interspike interval durations in the putamen were highly variable as evidenced by the large skew in interspike interval histograms (Fig. 4) and the irregular discharge pattern in the rate plots. The variability of interval size for each unit was assessed by calculating the coefficient of variation. The sample distributions of coefficients of variation were large and continuous in both groups (Fig. 5B,D), however, the proportion of

A

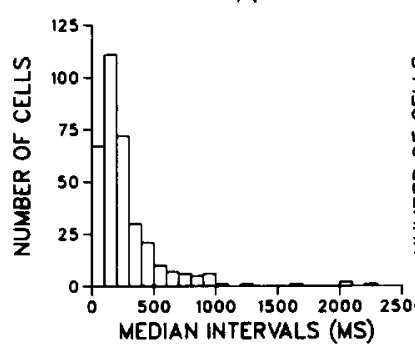

C
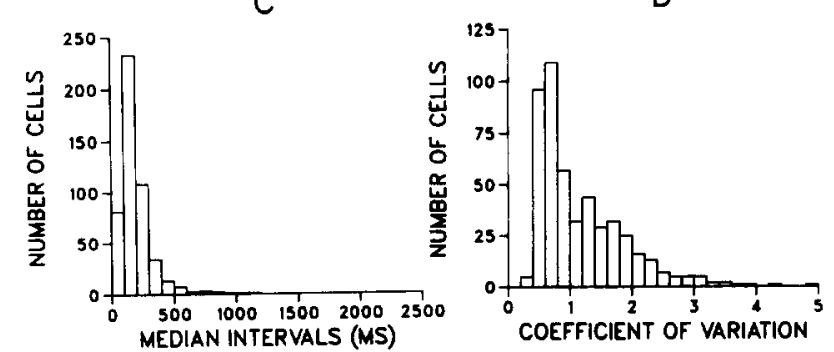

Fig. 6. The distributions of median intervals are plotted for control (A) and lesioned (C) animals. Median intervals in lesioned animals are significantly shorter than in controls. In A, 2 units exceeded boundaries of the $x$-axis and were not plotted. Plots B and D illustrate the distribution of coefficients of variation in control and lesioned animals, respectively. Variability was significantly greater in controls. units with low variability increased in the animals with cerebral cortical ablation. The median value of the coefficients of variation in lesioned animals (Table 1 ) was $63 \%$ of the value in controls. These changes are significant $(P<0.001)$.

Recording tracks were analyzed to determine if units with particular discharge properties were restricted to certain regions of the putamen. The sampling region for lesioned animals largely overlapped the region studied in controls (Fig. 2). No regional differences in median firing rates or coefficients of variation were detected in the lesioned animals or controls.

Caudate nucleus. Spontaneous discharge activity of units in the caudate nucleus was similar to that of units in the putamen (Table I). The notable difference in the caudate nucleus was the absence of a significant change following cortical ablation. Units in the caudate nucleus

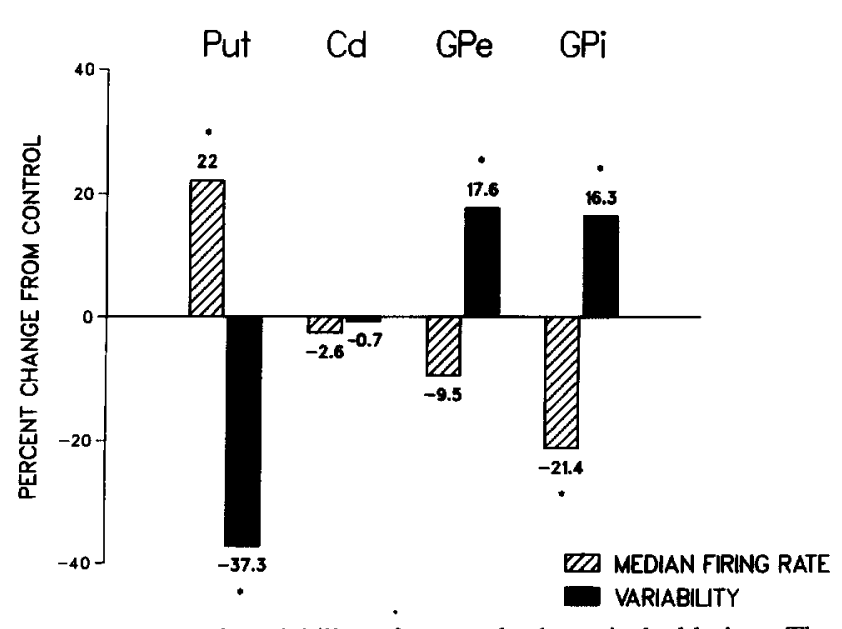

Fig. 7. Rate and variability after cerebral cortical ablation. The changes (percentage) from control values in the median firing rate (open bars) and the coefficient of variation (hatched bars) were computed and plotted for each structure. Decreases give negative values, Put, putamen; $\mathrm{Cd}$, caudate nucleus; GPe, globus pallidus external segment; GPi, globus pallidus internal segment. The asterisks indicate significant changes. 
A CONTROL

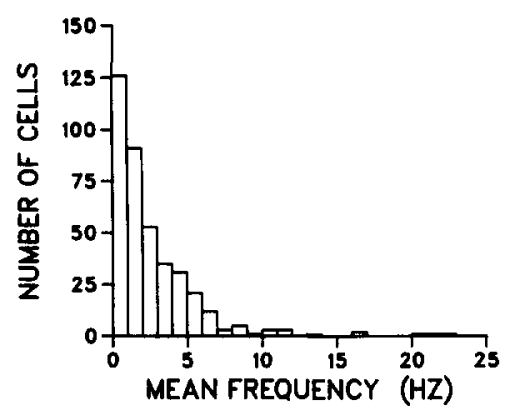

B LESION

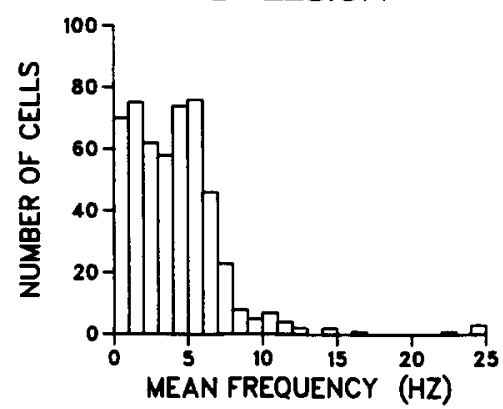

Fig. 8. The distributions of putaminal mean firing rates in spikes per second $(\mathrm{Hz})$ are plotted for control animals $(\mathrm{A})$ and lesioned animals (B). Three cells in the lesion group exceeded the boundaries of the $x$-axis and were not plotted. In the control group, $69 \%$ of the cells are found in the first 3 bins $(<3 \mathrm{~Hz})$ compared to $40 \%$ in the lesion group.

displayed no difference in the median interval (discharge rate) or the coefficients of variation in animals with cortical ablation compared to controls (Table I, Fig. 7).

Active cell density. All tracks through the neostriatum contained regions with spontaneous firing (active regions) interspersed with regions without spontaneous activity (inactive regions). The inactive regions contained silent neurons that on occasion fired briefly to the mechanical stimulation of electrode movement. Now and then, electrode movement would damage quiet cells, producing a characteristic injury discharge followed by silence. Similar distributions of active and inactive regions and mechanically activated units were found in

TABLE II

Active cell density

\begin{tabular}{lll}
\hline & Control & Lesion \\
\hline Putamen & & \\
$\quad$ Tracks & 21 & 25 \\
$\quad$ Units/mm (mean (S.D.)) & $3.8(2.1)$ & $4.3(1.6)$ \\
Caudate nucleus & 14 & 10 \\
$\quad$ Tracks & 14 & 10 \\
Units/mm (mean (S.D.)) & $4.0(3.0)$ & $3.1(0.7)$ \\
\hline
\end{tabular}

both control and lesioned animals. To assess the possibility that the sizes of the active regions in the neostriatum might change with the lesion, we estimated the relative incidence of active cells per millimeter of electrode track. Cells were counted from the first to the last cell encountered in each track within the caudate nucleus or the putamen and the distance between these points was determined. The results are summarized in Table II. The incidence of active cells did not differ significantly between control and lesioned animals in either the putamen or caudate nucleus.

Globus pallidus. In the control animals, spontaneous discharge activity in the globus pallidus was faster and less variable than in the neostriatum (Table I). After the ablation, units in the external segment of the globus pallidus discharged with longer intervals, i.e., with a decrease in rate (Table I, Fig. 7), though the change was not significant. Coupled with the decrease in rate, there was a significant increase in the variability of interval size (Table I, Fig. 7). In the internal segment, interval size increased significantly, i.e., there was a significantly slower discharge rate (Table I, Fig. 7). In the internal segment there was also a significant increase in the variability of interval size (Table I, Fig. 7) after the ablation.

\section{Recovery}

In order to identify any indication of recovery of neuronal activity from the effects of the cortical lesion, we examined the median interval and coefficient of variation as a function of time (number of days) after the lesion. No change in either property in any structure was evident over the 60 day study period.

\section{Lesion effects on motor behavior}

The effects of ablation of areas 4 and 6 in the monkey have been described in detail previously ${ }^{12,24}$. Briefly, there was a marked right hemiparesis that was apparent when the animals awakened from anesthesia. The affected leg was used for standing and made some forward progression movements with walking, but the arm was not used. Gradual improvement occurred and, by the fourth week, the right hindlimb was used more effectively in walking and the right arm developed some reaching movements. By the eighth week, the right leg regularly participated in walking and the right arm was used intermittently. The animals usually reached for objects with the left limbs, although the right arm occasionally made associated incomplete extension movements.

\section{DISCUSSION}

Single units in the neostriatum discharged spontane- 
ously with widely ranging and highly variable firing rates. The distributions of both discharge rate and variability were broad and continuous. Ablating cerebral cortical areas 4 and 6 produced a marked change in the discharge properties in the putamen. The most distinguishable difference was in the variability of firing, which was greatly reduced. In addition, the units discharged with a small but significant increase in median firing rate. In contrast, units in the caudate nucleus, which exhibit discharge properties similar to units in the putamen, did not demonstrate any significant change after the cerebral cortical lesion. In the globus pallidus, the median firing rate decreased after the lesion, although the change was significant only in the internal segment. The variability of firing increased significantly in both segments, and like the putamen, the changes in variability were greater than the changes in rate.

The findings in this study lead us to conclude that the precentral region of the cerebral cortex strongly influences the spontaneous discharge properties of units in the basal ganglia. While the cerebral cortex has some effect upon the tonic firing rate of these units, the greatest influence is upon the variability of discharge. Ablation of the precentral area of cortex, which has a devastating impact on motor function, would also have a major influence on information processing in the corticostriatal motor pathway. The results of the present experiment suggest that the variation, or pattern, of unit activity may be a more important encoding mechanism utilized in this circuit than discharge rate.

Unit firing rates in the putamen following the cortical deafferentation were regulated close to, or slightly faster, than control values. A number of mechanisms can be proposed to explain this finding. Putaminal units may regulate their own firing rates. Self-regulation of firing rates has been observed in the deafferented dentate gyrus, which displays an initial depression that gradually recovers and stabilizes over a period of about 8 days $^{52}$. Since no recordings were made in the 7 day post-surgical recovery period in the present study it is unknown whether this may have occurred. Plastic changes in neurotransmitter receptor properties ${ }^{66,67}$ may also contribute. Deafferentation in the CNS usually leads to an increase in receptor density and supersensitivity to neurotransmitters. Although receptors provide a potential site for intrinsic regulation following cortical deafferentation ${ }^{15,45,71}$ there is presently no evidence to indicate that this mechanism operates in this pathway.

A simple explanation for the persistence of putaminal activity after the lesion is that the restricted cortical ablation leaves intact a sufficiently large convergent input from subcortical areas and other cortical regions. The fact that variability of discharge decreases after the lesion suggests that the putamen receives its dynamic modulation from areas 4 and 6 , a view consistent with the important role that the putamen has in processing motor cortical inputs. Circuit architecture may be factor in this mechanism. In the striatum, cerebral cortical and thalamic afferents make synaptic contacts on the heads of spines along distal dendritic branches of medium spiny neurons ${ }^{17,19,32}$. Inhibitory terminals from neighboring neurons, on the other hand, contact the spine necks, proximal dendritic shafts and cell bodies ${ }^{17,19}$. The location of the inhibitory inputs gives them the possibility of gating or restricting the distal excitatory inputs from accessing the soma and the spike initiation zone. Even a continuous barrage of excitatory input could be blocked from activating the neuron. This mechanism may contribute to the low spontaneous discharge rate ${ }^{68}$ and may also explain the variable pattern of discharge. For example, focused cerebral activation, as observed in humans in recordings of cerebral blood flow during behavior $^{54,55}$ may increase striatal discharge. However, activity sufficient to overcome the proximal inhibition would probably induce additional activity in local circuits as well and thus, a discharge pattern in which a burst of spikes is quenched after a short delay by local inhibitory interactions is predicted. This type of discharge pattern would exhibit high variability such as we observed in the present study. Local processing would have an important role in regulating information flow in this type of network, and rate coding of information would be less important than pattern coding. Cortical afferents would have an important role in modulating neostriatal activity, but a partial loss of cortical inputs would not preclude postlesion tonic activity induced by remaining afferents and diminished local inhibition.

The above mechanism depends to a large degree on convergence in the neostriatum. While a restricted lesion leaves a sufficient number of afferents intact to maintain firing, larger lesions may remove so many afferent terminals that the units would cease to discharge. This could explain why large frontal cortex ablations in cats reduced the discharge rate in the neostriatum ${ }^{21,38}$ in contrast to the findings in the present study. The projection of neurons in the frontal cortex to the neostriatum is more widespread than the projections from the motor cortex ${ }^{61}$ and thus, a larger lesion would deafferent a larger number of terminals on each neuron. Other methodological differences between these studies and the present one also exist. Recordings were made in one session in the cats while the animals were immobilized in a holding frame and artificially respired. In contrast, the monkeys in the present study were recorded over many sessions and they were awake and loosely restrained in a seated position. 
Increased putaminal discharge activity after the ablation was unexpected. Our initial hypothesis that removing glutamatergic, presumably excitatory, cerebral cortical striatal afferents would decrease spontaneous firing must be rejected. In the pallidum, increased striatal discharge should inhibit activity through the GABAergic striatopallidal projection, and indeed, this change was observed. Our previous studies of striatal lesions demonstrated an increase in pallidal firing rate ${ }^{57}$ which is in keeping with this conceptual scheme. In no instance, however, were the changes in median discharge rate very large in comparison with the variability of firing. We conclude that a change in the variability of neuronal discharge is the best index of a change in afferent regulation.

Motor cortical afferents project primarily to the matrix compartment in the putamen ${ }^{23}$. Compartmentalized responses to cortical ablations can be observed in the differential expression of preproenkephalin and preprodynorphin mRNAs ${ }^{64}$. It is unknown, however, whether the physiological effects of these ablations are restricted to single compartments. Neighboring compartments may have physiological interactions through interneuronal contacts. In addition, indirect interactions might arise by afferents from other cortical or subcortical regions affected by the motor cortical lesion. Pallidal activity, for example, would be affected both by changes in striatal afferents and changes in afferents from the subthalamus, which receives a substantial projection from the motor cortex ${ }^{9}$. The increased variability observed in the pallidum after the cortical lesion may be due to an interaction in these convergent pathways. In this study, compartmental responses were restricted to a differential bias towards the putamen and an irregular distribution of active units. Functional clustering has been observed physiologically ${ }^{4}$. Whether the irregular distribution might be related in some way to differential activity in the patch and matrix compartments ${ }^{28,30}$ remains unknown. It is possible that an underlying compartmental effect in the neostriatum, inaccessible to our measures of neuronal activity, may produce the differential effects on the two segments of the globus pallidus. Striatal lesions have been shown to produce differential changes in the pallidum ${ }^{53,57}$.

There are physiological data to support striatal compartmentalization at the level of single units ${ }^{4,31,33,34}$. For example, Kimura ${ }^{33}$ demonstrated two unit categories classified in part by firing rate, type I (tonically firing) and type II (slowly firing), making up $41 \%$ and $59 \%$ of a striatal sample. With this rate classification, our control sample yields similar proportions, $31 \%$ and $69 \%$, for types I and II, respectively. It is not known whether the similarity extends to behavioral response characteristics, however, since the priority of our data collection was to sample a unit population without restriction to a behavioral task. The decrease in type II units after the lesion suggests that units may convert from one type to another depending on the cortical input to the neuron. Other studies have described striatal units in different proportions ${ }^{4,31}$. Comparing unit types between studies selected with a particular task to spontaneously active units or to units performing different tasks is difficult and inconsistent. The continuous distributions of firing rates and variability did not lend themselves to obvious categorization schemes and required arbitrary divisions for comparisons. Furthermore, mean rate classifications are complicated by the fact that the interval distributions are highly skewed. Even a few long intervals bias the arithmetic mean toward low rates, greatly exaggerating the difference in firing rates between control and lesioned animals. Median interval is a better choice for comparisons where the data exhibit skewed distributions ${ }^{16}$ such as those produced by single unit discharge. It is possible that neurons other than the ubiquitous medium spiny neuron may produce a particular discharge pattern or 'type', for example cholinergic neurons, but even if they had a distinctive discharge signature, their small numbers may not make them easily distinguishable in data with wide distributions.

The observed changes in unit activity could not be attributed to non-specific surgical effects such as trauma or edema. Putamen neurons, which receive larger numbers of afferents from areas 4 and 6 , were more affected by the ablation than were caudate nucleus neurons, supporting the idea that the changes in unit activity were due to specific effects of the lesion. The caudate nucleus, which lies directly underneath the lesion site, would be more likely to exhibit non-specific surgery-induced changes than the putamen. Nevertheless, the caudate nucleus showed no significant change in neuronal unit activity. A similar pattern of relatively preserved activity in the caudate nucleus associated with striking changes in the putamen has been observed with metabolic mapping ${ }^{12}$. Moreover, it has been shown that the deficit in metabolic activity in the putamen persists for two months following this lesion. In contrast, the caudate nucleus recovers significantly in this time ${ }^{24}$.

Hemiparesis in the lesioned animals precluded the use of a sensory-motor task in this experiment. The importance of using awake animals free from anesthetics for studies of spontaneous discharge properties has been verified $^{20}$ especially in the basal ganglia ${ }^{48}$. Our goal was to record spontaneous activity during quiet sitting conditions, simulating earlier metabolic studies ${ }^{12,24}$. In order to reject spike trains associated with adventitious movements, we opted for a technique to monitor movements. 
We rejected the use of electromyographic recordings because it was not technically feasible to record continuously from all of the muscles that could potentially be activated. In spite of the fact that the animals generally sat quietly and our analysis strived to eliminate all periods of overt motor activity from the recordings selected for assessment, some postural movements may have escaped detection. We believe, however, that undetected movements can not explain our observations. The experimental setting did not produce the conditions to cause repetitive sensory-triggered, well-learned movements typically found to activate basal ganglia neurons. Moreover, it has been shown in comparisons of conditioned movements to spontaneous postural movements that only the former are correlated with striatal discharge $e^{42,56,60}$. Positron emission tomography studies in humans also demonstrate that striatal activation correlates best with overlearned, semiautomatic limb movements compared to novel limb movements ${ }^{43}$. Faster firing rates in the lesioned animals also support the idea that the activity differences observed were due to the lesion and not undetected movements. Most studies ${ }^{1,3,5-7}$,

\section{REFERENCES}

1 Aldridge, J.W., Anderson, R.J. and Murphy, J.T., Sensory motor processing in the caudate nucleus and globus pallidus: a single unit study in behaving primates, Can. J. Physiol. Pharmacol., 58 (1980) 1192-1201.

2 Aldridge, J.W., Walden, J.L. and Gilman, S., Enhancing high speed digitization of single unit neuronal activity on a microcomputer using a hybrid software-hardware technique, J. Neurosci. Methods, 28 (1989) 205-208.

3 Alexander, G.E., Selective neuronal discharge in monkey putamen reflects intended direction of planned limb movements, Exp. Brain Res., 67 (1987) 623-634.

4 Alexander, G.E. and DeLong, M.R., Microstimulation of the primate neostriatum. II. Somatotopic organization of striatal microexcitable zones and their relation to neuronal response properties, J. Neurophysiol., 53 (1985) 1417-1430.

5 Allum, J.H.J., Anner-Baratti, R.E.C. and Hepp-Reymond, M.C., Activity of neurons in the 'motor' thalamus and globus pallidus during the control of isometric finger force in the monkey, Exp. Brain Res., Suppl. 7 (1983) 194-203.

6 Anderson, M.E., Discharge patterns of basal ganglia neurons during active maintenance of postural stability and adjustment to chair tilt, Brain Research, 143 (1977) 325-338.

7 Anderson, M.E. and Horak, F.B., Influence of the globus pallidus on arm movements in monkeys. III. Timing of movement-related information, J. Neurophysiol., 54 (1985) 433-448.

8 Beckstead, R.M. and Cruz, C.J., Striatal axons to the globus pallidus, entopeduncular nucleus and substantia nigra come mainly from separate cell populations in the cat, Neuroscience, 19 (1986) 147-158.

9 Carpenter, M.B., Anatomy of the corpus striatum and brain stem integrating systems. In V. Brooks (Ed.), Handbook of Physiology. Section 1. Nervous System, Vol. 2, Motor Control, Williams and Wilkins, Baltimore, 1981, pp. 947-996.

10 Crutcher, M.D. and DeLong, M.R., Single cell studies of the primate putamen. I. Functional organization, Exp. Brain Res., 53 (1984) 233-243.

11 Crutcher, M.D. and DeLong, M.R., Single cell studies of the
$10,11,13,22,31,34,39,58,59$ indicate a predominance of excitatory activity associated with motor behavior, which is in keeping with the normal low rate of spontaneous striatal discharge. Since the present study demonstrated increased discharge activity in the partially deafferented striatum in animals unable to make limb movements, it seems unlikely that undetected movements will explain the differences in firing pattern and rate. While the possibility exists that some of the unit activity, which consists of several hundred recordings made in many separate recording sessions from four animals, may have been modulated by movements, it is highly improbable that a systematic movement bias could account for the difference in discharge pattern between control and lesioned animals.

Acknowledgements. The authors acknowledge the technical assistance of: M. Gallagher, D. Jaeger, M. Lesperance, S. Ruger, R. Sachdev, M. Samson, and J. Walden and helpful comments on the manuscript from D. Jaeger and R. Sachdev. This work was supported in part by NIH Grants NS19613 and NS07222 and by a grant from the United Cerebral Palsy Research and Educational Foundation, Inc.

primate putamen. II. Relations to direction of movement and pattern of muscular activity, Exp. Brain Res., 53 (1984) 244-258.

12 Dauth, G.W., Gilman, S., Frey, K.A. and Penney Jr., J.B., Basal ganglia glucose utilization after recent precentral ablation in the monkey, Ann. Neurol., 17 (1985) 431-438.

13 DeLong, M.R., Crutcher, M.D. and Georgopoulos, A.P., Primate globus pallidus and subthalamic nucleus: functional organization, J. Neurophysiol., 53 (1985) 530-543.

14 Denny-Brown, D., The Cerebral Control of Movement, Liverpool University Press, Liverpool, 1966.

15 Divac, I., Fonnum, F. and Storm-Mathisen, J., High affinity uptake of glutamate in terminals of corticostriatal axons, Nature (Lond.), 266 (1977) 377-378.

16 Downie, N.M. and Heath, R.W., Basic Statistical Methods, Harper and Row, New York, 1974.

17 Dube, L., Smith, A.D. and Bolam, J.P., Identification of synaptic terminals of thalamic or cortical origin in contact with distinct medium-size spiny neurons in the rat neostriatum, $J$. Comp. Neurol., 257 (1988) 455-471.

18 Emson, P.C., Arregui, A., Clement-Jones, V., Sandberg, B.E.B. and Rossor, M., Regional distribution of methionineenkephalin and substance P-like immunoreactivity in normal human brain and in Huntington's disease, Brain Research, 199 (1980) 147-160.

19 Freund, T.F., Powell, J.F. and Smith, A.D., Tyrosine hydroxylase-immunoreactive boutons in synaptic contact with identified striatonigral neurons, with particular reference to dendritic spines, Neuroscience, 13 (1985) 1189-1216.

20 Fujiwara, N., Higashi, H., Nishi, S., Shimoji, K., Sugita, S. and Yoshimura, M., Changes in spontaneous firing patterns of rat hippocampal neurones induced by volatile anaesthetics, $J$. Physiol. (Lond.), 402 (1988) 155-176.

21 Garcia-Rill, E., Hull, C.D., Levine, M.S. and Buchwald, N.A., The spontaneous firing patterns of forebrain neurons. IV. Effects of bilateral and unilateral frontal cortical ablations on firing of caudate, globus pallidus and thalamic neurons, Brain Research, 165 (1979) 23-36.

22 Georgopoulos, A.P., DeLong, M.R. and Crutcher, M.D., Relations between parameters of step-tracking movements and 
single cell discharge in the globus pallidus and subthalamic nucleus of the behaving monkey, $J$. Neurosci., 3 (1983) 15861598.

23 Gerfen, C.R., The neostriatal mosaic: the reiterated processing unit. In M. Sandler, C. Feuerstein and B. Scatton (Eds.), Neuratransmitter Interactions in the Basal Ganglia, Raven, New York, 1987, pp. 19-29.

24 Gilman, S.,Dauth, G.W., Frey, K.A. and Penney Jr., J.B., Experimental hemiplegia in monkey: basal ganglia glucose activity during recovery, Ann. Neurol., 22 (1987) 370-376.

25 Gilman, S., Lieberman, J.S. and Marco, L.A., Spinal mechanisms underlying the effects of unilateral ablation of areas 4 and 6 in monkeys, Brain, 97 (1974) 49-64.

26 Gilman, S. and Marco, L.A., Effects of medullary pyramidotomy in the monkey. I. Clinical and electromyographic abnormalities, Brain, 94 (1971) 495-514.

27 Goldman, P.S. and Nauta, W.J.H., An intricately patterned prefronto-caudate projection in the rhesus monkey, J. Comp. Neurol., 171 (1977) 369-386.

28 Graybiel, A.M., Neurochemically specified subsystems in the basal ganglia. In Functions of the Basal Ganglia, Ciba Foundation Symposium 107, Pitman, London, 1984, pp. 114-119.

29 Graybiel, A.M., Neuropeptides in the basal ganglia. In J.B. Martin and J.D. Barchas (Eds.), Neuropeptides in Neurologic and Psychiatric Disease, Raven, New York, 1986, pp. 135-161.

30 Graybiel, A.M. and Ragsdale, C.W., Histochemically distinct compartments in the striatum of human, monkey, and cat demonstrated by acetylcholinesterase staining, Proc. Natl. Acad. Sci. U.S.A., 75 (1978) 5723-5726.

31 Hikosaka, O., Sakamoto, M. and Usui, S., Functional properties of monkey caudate neurons. 1 . Activities related to saccadic eye movements, J. Neurophysiol., 61 (1989) 780-798.

32 Kemp, J.M. and Powell, T.P., The site of termination of afferent fibres in the caudate nucleus, Philos. Trans. R. Soc. Lond. Biol. Sci., 262 (1971) 413-427.

33 Kimura, M., The role of primate putamen neurons in the association of sensory stimuli with movement, Neurosci. Res., 3 (1986) 436-443.

34 Kimura, M., Rajkowski, J.R. and Evarts, E., Tonically discharging putamen neurons exhibit set-dependent responses, Proc. Natl. Acad. Sci. U.S.A., 81 (1984) 4998-5001.

$35 \mathrm{Kita}, \mathrm{H}$. and Kitai, S.T., Glutamate decarboxylase immunoreactive neurons in rat neostriatum: their morphological type and populations, Brain Research, 447 (1988) 346-359.

$36 \mathrm{Künzle,} \mathrm{H.,} \mathrm{Bilateral} \mathrm{projections} \mathrm{from} \mathrm{precentral} \mathrm{motor} \mathrm{cortex}$ to the putamen and other parts of the basal ganglia. An autoradiographic study in Macaca fascicularis, Brain Research, 88 (1975) 195-209.

37 Levine, M.S., Hull, C.D. and Buchwald, N.A., Pallidal and entopeduncular intracellular responses to striatal, cortical, thalamic, and sensory inputs, Exp. Neurol., 44 (1974) 448-460.

38 Levine, M.S., Hull, C.D., Villablanca, J.R., Buchwald, N.A. and Garcia-Rill, E., Effects of caudate nuclear or frontal cortical ablation in neonatal kittens or adult cats on the spontaneous firing of forebrain neurons, Dev. Brain Res., 4 (1982) 129-138.

39 Liles, S.L., Activity of neurons in putamen during active and passive movements of wrist, J. Neurophysiol., 53 (1985) 217236.

40 Loopuijt, L.D. and Van der Kooy, D., Organization of the striatum: collateralization of its efferent axons, Brain Research, 348 (1985) 86-99.

41 Malliani, A. and Purpura, D.P., Intracellular studies of the corpus striatum. II. Patterns of synaptic activities in lenticular and entopeduncular neurons, Brain Research, 6 (1967) 341-354.

42 Manetto, C. and Lidsky, T.I., Caudate neuronal activity in cats during head turning: selectivity for sensory-triggered movement, Brain Res. Bull., 166 (1986) 425-428.

43 Mazziotta, J.C., Phelps, M.E. and Wapenski, J.A., Human cerebral motor system metabolic responses in health and disease, J. Cereb. Blood Flow Metabol., 5 (1985) S213-\$214.
44 McGeer, E.G., Staines, W.A. and McGeer, P.L., Neurotransmitters in the basal ganglia, Can. J. Neurol. Sci., 11 (1984) 89-99.

45 McGeer, P.L., McGeer, E.G., Scherer, U. and Singh, K., A glutamatergic corticostriatal path?, Brain Research, 128 (1977) 369-373.

46 Okoyama, S., Nakamura, Y., Moriizumi, T. and Kitao, Y., Electron microscopic analysis of the synaptic organization of the globus pallidus in the cat, J. Comp. Neurol., 265 (1987) 323-331.

47 Pan, H.S., Frey, K.A., Young, A.B. and Penney Jr., J.B., Changes in $\left[{ }^{3} \mathrm{H}\right]$ muscimol binding in substantia nigra, entopeduncular nucleus, globus pallidus, and thalamus after striatal lesions as demonstrated by quantitative receptor autoradiography, J. Neurosci., 3 (1983) 1189-1198.

48 Pan, H.S. and Walters, J.R., Unilateral lesion of the nigrostriatal pathway decreases the firing rate and alters the firing pattern of globus pallidus neurons in the rat, Synapse, 2 (1988) 650-656.

49 Park, M.R., Falls, W.M. and Kitai, S.T., An intracellular HRP study of the rat globus pallidus. I. Responses and light microscopic analysis, J. Comp. Neurol., 211 (1982) 284-294.

50 Penny, G.R., Afsharpour, S. and Kitai, S.T., The glutamate decarboxylase-, leucine enkephalin-, methionine enkephalin-, and substance P-immunoreactive neurons in the neostriatum of the rat and cat: evidence for partial population overlap, Neuroscience, 17 (1986) 1011-1045.

51 Preston, R.J., Bishop, G.A. and Kitai, S.T., Medium spiny neuron projection from the rat striatum: an intracellular horseradish peroxidase study, Brain Research, 183 (1980) 253-263.

52 Reeves, T.M. and Steward, O., Changes in the firing properties of neurons in the dentate gyrus with denervation and reinnervation: implications for behavioral recovery, Exp. Neurol., 102 (1988) 37-49.

53 Reiner, A., Albin, R.L., Anderson, K.D., D'Amato, C.J., Penney, J.B. and Young, A.B., Differential loss of striatal projection neurons in Huntington's disease, Proc. Natl. Acad. Sci. U.S.A., 85 (1988) 5733-5737.

54 Roland, P.E., Different cortical areas in man in organization of voluntary movements in extrapersonal space, $J$. Neurophysiol., 43 (1980) 137-150.

55 Roland, P.E., Cortical organization of voluntary behavior in human, Human Neurobiol., 4 (1985) 155-168.

56 Rolls, E.T., Thorpe, S.J. and Maddison, S.P., Responses of striatal neurons in the behaving monkey. I. Head of the caudate nucleus, Behav. Brain Res., 7 (1983) 179-210.

57 Sachdev, R.N.S., Gilman, S. and Aldridge, J.W., Effects of excitotoxic striatal lesions on single unit activity in globus pallidus and entopeduncular nucleus of the cat, Brain Research, 507 (1989) 295-306.

58 Schneider, J.S. and Lidsky, T.I., Processing of somatosensory information in striatum of behaving cats, $J$. Neurophysiol., 45 (1981) 841-851.

59 Schneider, J.S., Morse, J.R. and Lidsky, T.I., Somatosensory properties of globus pallidus neurons in awake cats, Exp. Brain Res., 46 (1982) 311-314.

60 Schultz, W. and Romo, R., Neuronal activity in the monkey striatum during the initiation of movements, Exp. Brain Res., 71 (1988) 431-436.

61 Selemon, L.D. and Goldman-Rakic, P.S., Longitudinal topography and interdigitation of corticostriatal projections in the Rhesus monkey, J. Neurosci., 5 (1985) 776-794.

62 Smith, Y. and Parent, A., Differential connections of caudate nucleus and putamen in the squirrel monkey (Saimiri sciureus), Neuroscience, 18 (1986) 347-372.

63 Smith, Y., Parent, A., Seguela, P. and Descarries, L., Distribution of GABA-immunoreactive neurons in the basal ganglia of the squirrel monkey (Saimiri sciureus), J. Comp. Neurol., 259 (1987) 50-64.

64 Uhl, G.R., Navis, B. and Douglas, J., Differential expression of preproenkephalin and preprodynorphin mRNAs in striatal neurons: high levels of preproenkephalin expression depend on 
cerebral cortical afferents, J. Neurosci., 8 (1988) 4755-4764. 65 Vibert, J.F. and Costa, J., Spike separation in multiunit records: a multivariate analysis of spike descriptive parameters, $E E G$ Clin. Neurophysiol., 47 (1979) 172-182.

66 Waddington, J.L. and Cross, A.J., Denervation supersensitivity in the striatonigral GABA pathway, Nature (Lond.), 276 (1978) 618-620.

67 Waddington, J.L. and Cross, A.J., Characterization of denervation supersensitivity in the striatonigral GABA pathway of the kainic acid-lesioned rat and in Huntington's disease, Brain Res. Bull., 5 (1980) 825-828.

68 Wilson, C.J., Chang, H.T. and Kitai, S.T., Disfacilitation and long-lasting inhibition of neostriatal neurons in the rat, Exp. Brain Res., 51 (1983) 227-235.

69 Wilson, C.J. and Phelan, K.D., Dual topographic representation of neostriatum in the globus pallidus of rats, Brain Research, 243 (1982) 354-359.

70 Yeterian, E.H. and Van Hoesen, G.W., Cortico-striate projections in the rhesus monkey: the organization of certain corticocaudate connections, Brain Research, 139 (1978) 43-63.

71 Young, A.B., Bromberg, M.B. and Penney Jr., J.B., Decreased glutamate uptake in subcortical areas deafferented by sensorimotor cortical ablation in the cat, J. Neurosci., 1 (1981) 241-249. 